\title{
Mechanical Stress Behavior Inside Slots of Large Hydro Generators
}

\author{
C. Grabner and H. Köfler \\ Institute of Electrical Machines and Drives \\ Graz University of Technology \\ Kopernikusgasse 24, A-8010 Graz (Austria) \\ phone:+43 316873 8602, fax:+43 316873 8103, e-mail: grabner@ema.tu-graz.ac.at, koefler@ema.tu-graz.ac.at
}

\begin{abstract}
The mechanical design and construction of slot wedges which are typically used in large synchronous machines must be robust enough to resist all occurring electromagnetic and mechanical force magnitudes within various operational states. Therefore, the invoked mechanical stress distributions near the slot wedge and the slot bottom are investigated with the aid of numerical and analytical methods. All performed investigations are carried out for several material properties which are typically used for industry applications.
\end{abstract}

\section{Key words}

Electromagnetic force densities, Magnetic saturation, Anisotropic materials, Electromechanical interaction, Finite element analysis.

\section{Introduction}

The structure of the upper and the lower Roebel bar itself consists of sub conductors, insulation, middle filler piece and envelope [1]. Some details of the investigated Roebel bar construction are depicted in Fig. 1.

The cross section of the investigated Roebel bars with the included several filling materials inside the slot is shown in Fig. 2.

A slot wedge fixes all material parts inside the slot and avoids any free space in radial direction. The computation of the mechanical stress distributions along the slot wedge and the slot bottom of Fig.2 is of main interest in our investigations. The Table I contains the characteristic dimensions of the investigated slot structure.

The nonlinear magnetic saturation effects and anisotropic mechanical material properties are fully considered in the proposed numerical investigation. Unfortunately, the enormous long solving time and the high memory requirement are the main disadvantages arising with the numerical solution process.

Thus, the coupled electromechanical problem is additionally solved in an analytical way. But the prediction of the governing mechanical quantities due to the proposed analytical method is based on simplifying assumptions. Thereby, the electromagnetic material properties, such as e.g. the nonlinearities or the mechanical anisotropic behavior are regarded with introduced coefficients.

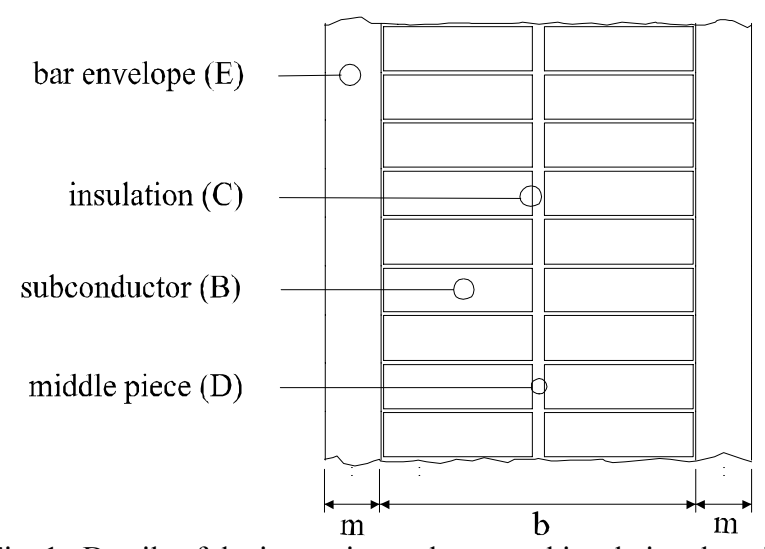

Fig. 1. Details of the isotropic conductor and insulation domain enclosed by the mainly anisotropic bar envelope.

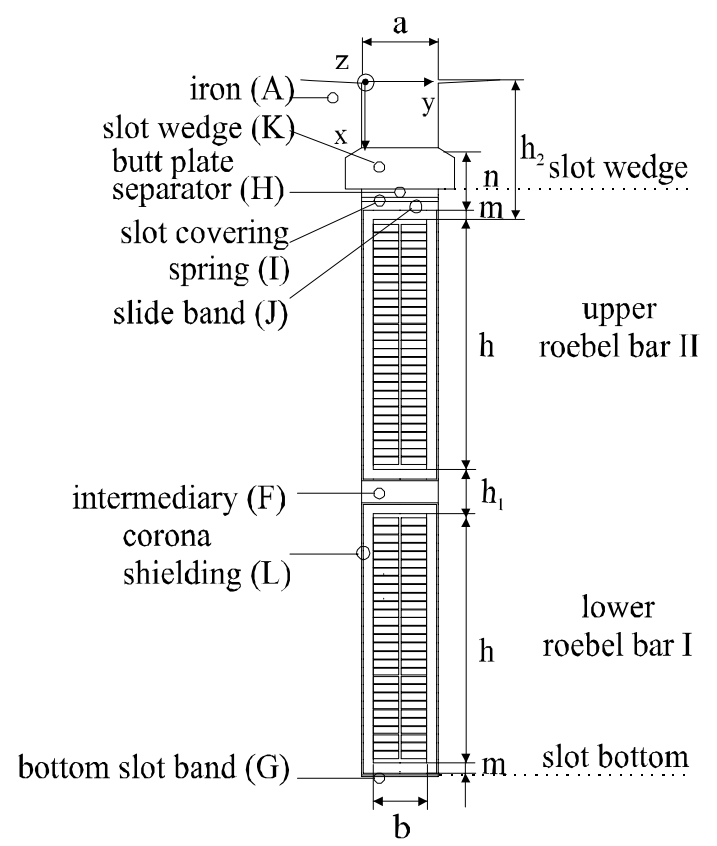

Fig. 2. Composite materials embedded inside the slot.

TABLE I. - Characteristic dimensions of Roebel bar and slot

\begin{tabular}{lccc}
\hline Rated current & $\mathrm{I}_{\mathrm{N}}$ & 3888 & $\mathrm{~A}$ \\
Width of conducting region & $\mathrm{b}$ & 0.0159 & $\mathrm{~m}$ \\
Height of conductor region & $\mathrm{h}$ & 0.0709 & $\mathrm{~m}$ \\
Height of intermediary region & $\mathrm{h}_{1}$ & 0.0155 & $\mathrm{~m}$ \\
Height of top region & $\mathrm{h}_{2}$ & 0.0414 & $\mathrm{~m}$ \\
Thickness of bar envelope & $\mathrm{m}$ & 0.0031 & $\mathrm{~m}$ \\
Height of slot wedge region & $\mathrm{n}$ & 0.0168 & $\mathrm{~m}$ \\
Slot width & $\mathrm{a}$ & 0.0220 & $\mathrm{~m}$ \\
\hline
\end{tabular}




\section{The coupled electromechanical problem for anisotropic materials}

A. Occurring volume as well as surface forces of electromagnetic origin

The steady electromagnetic field inside a closed volume is described with the subset of Maxwell equations

$$
\begin{gathered}
\vec{\nabla} \cdot \vec{B}=0, \\
\vec{\nabla} \times \vec{H}=\vec{J},
\end{gathered}
$$

and the material relationship

$$
\mu \vec{H}=\vec{B},
$$

which takes account of the nonlinear magnetic material properties $[2,3]$. The boundary conditions of the electromagnetic field problem (1) to (3) are given as [4]

$$
\vec{n} \cdot \vec{B}=0 \text {, }
$$

or alternatively with

$$
\vec{n} \times \vec{H}=\overrightarrow{0} .
$$

The acting volume force density inside the considered conducting domains with an assumed constant relative permeability across the copper regions and additionally occurring volume force density due to the varying local relative permeability values within magnetic nonlinear materials are given with [5]

$$
\vec{f}=\vec{J} \times \vec{B}-\frac{1}{2}(\vec{H} \cdot \vec{H}) \vec{\nabla} \mu .
$$

Force components due to the magnetostrictive effects in iron materials are omitted in (6). A rewritten form of (6) is given with the identity

$$
(\vec{\nabla} \times \vec{H}) \times \vec{H}=(\vec{\nabla} \otimes \vec{H}) \cdot \vec{H}-\frac{1}{2} \vec{\nabla}(\vec{H} \cdot \vec{H}),
$$

as

$$
\vec{f}=\mu \vec{H} \cdot(\vec{\nabla} \otimes \vec{H})-\mu \frac{1}{2} \vec{\nabla}(\vec{H} \cdot \vec{H})-\frac{1}{2}(\vec{H} \cdot \vec{H}) \vec{\nabla} \mu
$$

The introduction of a space dependent permeability as

$$
\vec{\nabla}(\mu(\vec{H} \cdot \vec{H}))=\mu \vec{\nabla}(\vec{H} \cdot \vec{H})+(\vec{H} \cdot \vec{H}) \vec{\nabla} \mu,
$$

and the application of

$$
\vec{\nabla} \cdot((\mu \vec{H}) \otimes \vec{H})=(\vec{\nabla} \cdot(\mu \vec{H})) \vec{H}+(\mu \vec{H}) \cdot(\vec{\nabla} \otimes \vec{H}),
$$

yields with the relation (8) to the expression

$$
\vec{\nabla} \cdot\left\{-\mu \frac{1}{2}(\vec{H} \cdot \vec{H}) \underset{\sim}{\delta}+\mu \vec{H} \otimes \vec{H}\right\}=\vec{f},
$$

whereby the abbreviation

$$
\underset{\sim}{p} p^{e}=\frac{1}{\mu} \vec{B} \otimes \vec{B}-\left(\frac{1}{2 \mu} B^{2}\right) \underset{\sim}{\delta}
$$

of the momentum flow density tensor is used [6,7].

The electromagnetic surface force density is given along a considered cutting surface through the volume with

$$
\vec{f}^{s}=-\llbracket \underset{\sim}{p^{e}} \rrbracket \cdot \vec{n} \text {. }
$$

Thereby, the useful abbreviation

$$
\llbracket \underset{\sim}{p}{\underset{\sim}{e}}^{e} \rrbracket={\underset{\sim}{p}}^{e,(2)}-{\underset{\sim}{p}}^{e,(1)}
$$

is defined as the difference of (12) obtained by an infinite approach to both sides of the considered surface [5].

\section{$B$. The interaction with the mechanical system}

The coupling between the two dimensional mechanical stress and the electromagnetic field is established with

$$
\vec{\nabla} \cdot \underset{\sim}{\sigma}=-\vec{f}
$$

whereby the electromagnetic volume forces (6) are used [8]. A unique mechanical boundary problem is defined by the default displacement components for the radial displacement part

$$
\vec{n} \cdot \vec{V}=u
$$

and for the tangential displacement part

$$
\vec{n} \times \vec{V}=v .
$$

The mechanical stress magnitude of a solid body which is enclosed by air domains is vanishing

$$
\tilde{\sim}^{(1)} \cdot \vec{n}=\overrightarrow{0}
$$

along the outer surface boundary, if no electromagnetic field quantities are present [9]. Alternatively, the mechanical surface force density

$$
\vec{f}^{s}=-\llbracket \underset{\sim}{\sigma} \rrbracket \cdot \vec{n}
$$

must be applied at the considered mechanical boundary. Similarly to (14), the expression (19) can be rewritten as

$$
\llbracket \underset{\sim}{\sigma} \rrbracket \cdot \vec{n}={\underset{\sim}{\sigma}}^{(2)} \cdot \vec{n}
$$

whereby the stress values (18) are disappearing. The distribution of the electromagnetic surface force density (13) is supposed to act as mechanical stress (19) along the material boundary of the solid body according to

$$
\llbracket \underset{\sim}{p} p^{e} \rrbracket \cdot \vec{n}={\underset{\sim}{\sigma}}^{(2)} \cdot \vec{n}
$$

The term (21) can be further rewritten by means of (12) and (14) as

$$
{\underset{\sim}{(2)}}^{(\vec{n}}=\frac{1}{2 \mu_{0}} \frac{\mu_{r}^{(1)}-\mu_{r}^{(2)}}{\mu_{r}^{(1)} \mu_{r}^{(2)}}\left(\vec{B}^{(1)} \cdot \vec{B}^{(2)}\right) \vec{n} .
$$

Thereby, the unique interrelation between the acting mechanical stresses on surrounding material surfaces and the saturation dependent relative permeability values as well as the saturation dependent magnetic flux densities on both sides of the boundary, indicated with the superscript (1) and (2), are taken into account. Hence, both saturation dependent electromagnetic quantities, namely the relative permeability and the magnetic flux density have a common influence on the magnitude of the acting surface force density (22) [10].

The interrelationship between the second order mechanical strain tensor

$\underset{\sim}{\varepsilon}=\varepsilon_{x x} \vec{e}_{x} \otimes \vec{e}_{x}+\varepsilon_{x y} \vec{e}_{x} \otimes \vec{e}_{y}+\varepsilon_{y x} \vec{e}_{y} \otimes \vec{e}_{x}+\varepsilon_{y y} \vec{e}_{y} \otimes \vec{e}_{y}$

and the second order mechanical stress tensor

$\underset{\sim}{\sigma}=\sigma_{x x} \vec{e}_{x} \otimes \vec{e}_{x}+\sigma_{x y} \vec{e}_{x} \otimes \vec{e}_{y}+\sigma_{y x} \vec{e}_{y} \otimes \vec{e}_{x}+\sigma_{y y} \vec{e}_{y} \otimes \vec{e}_{y}$

is established with

$$
\underset{\sim}{\sigma}=\underset{\sim}{c} \cdot \underset{\sim}{\mathcal{E}}
$$

and the fourths order material tensor $[8,11]$

$$
\begin{aligned}
\underset{\sim}{c} & =c_{x x x x} \vec{e}_{x} \otimes \vec{e}_{x} \otimes \vec{e}_{x} \otimes \vec{e}_{x}+c_{x x x y} \vec{e}_{x} \otimes \vec{e}_{x} \otimes \vec{e}_{x} \otimes \vec{e}_{y}+ \\
& +c_{x x y x} \vec{e}_{x} \otimes \vec{e}_{x} \otimes \vec{e}_{y} \otimes \vec{e}_{x}+c_{x x y y} \vec{e}_{x} \otimes \vec{e}_{x} \otimes \vec{e}_{y} \otimes \vec{e}_{y}+ \\
& +c_{x y x x} \vec{e}_{x} \otimes \vec{e}_{y} \otimes \vec{e}_{x} \otimes \vec{e}_{x}+c_{x y x y} \vec{e}_{x} \otimes \vec{e}_{y} \otimes \vec{e}_{x} \otimes \vec{e}_{y}+ \\
& +c_{x y y x} \vec{e}_{x} \otimes \vec{e}_{y} \otimes \vec{e}_{y} \otimes \vec{e}_{x}+c_{x y y y} \vec{e}_{x} \otimes \vec{e}_{y} \otimes \vec{e}_{y} \otimes \vec{e}_{y}+ \\
& +c_{y x x x} \vec{e}_{y} \otimes \vec{e}_{x} \otimes \vec{e}_{x} \otimes \vec{e}_{x}+c_{y x x y} \vec{e}_{y} \otimes \vec{e}_{x} \otimes \vec{e}_{x} \otimes \vec{e}_{y}+ \\
& +c_{y x y x} \vec{e}_{y} \otimes \vec{e}_{x} \otimes \vec{e}_{y} \otimes \vec{e}_{x}+c_{y x y y} \vec{e}_{y} \otimes \vec{e}_{x} \otimes \vec{e}_{y} \otimes \vec{e}_{y}+ \\
& +c_{y y x x} \vec{e}_{y} \otimes \vec{e}_{y} \otimes \vec{e}_{x} \otimes \vec{e}_{x}+c_{y y x y} \vec{e}_{y} \otimes \vec{e}_{y} \otimes \vec{e}_{x} \otimes \vec{e}_{y}+ \\
& +c_{y y y x} \vec{e}_{y} \otimes \vec{e}_{y} \otimes \vec{e}_{y} \otimes \vec{e}_{x}+c_{y y y y} \vec{e}_{y} \otimes \vec{e}_{y} \otimes \vec{e}_{y} \otimes \vec{e}_{y} .
\end{aligned}
$$


Numerical investigations require the determination of all coefficients which are occurring in (26) for each kind of composite material. Thereby, the equality of the mechanical shear stress $\sigma_{x y}=\sigma_{y x}$ allows writing each strain component in an anisotropic material as a linear function of three stress components,

$$
\begin{aligned}
& \varepsilon_{x x}=\lambda_{x x x x} \sigma_{x x}+\lambda_{x x y y} \sigma_{y y}+\lambda_{x x x y} \sigma_{x y}, \\
& \varepsilon_{y y}=\lambda_{y y x x} \sigma_{x x}+\lambda_{y y y y} \sigma_{y y}+\lambda_{y y x y} \sigma_{x y}, \\
& \varepsilon_{x y}=\lambda_{x y x x} \sigma_{x x}+\lambda_{x y y y} \sigma_{y y}+\lambda_{x y x y} \sigma_{x y} .
\end{aligned}
$$

The obvious nine unknown coefficients in (27) must be verified. The tensile and shear stresses inside an anisotropic material are schematically given in Fig.3.

Reinforced materials have mostly a certain kind of structural symmetry. Depending on the direction of embedded reinforcement layers there exist three mutually orthogonal planes of elastic symmetry which passes through each point of the solid body. Thus the coefficients

$$
\lambda_{x x x y}=\lambda_{y y x y}=\lambda_{x y x x}=\lambda_{x y y y}=0
$$

are disappearing in (27). Consequently, the generalized Hook's law (27) can be rewritten with the modulus of elasticity and the transverse contraction for an orthotropic anisotropy material as

$$
\begin{gathered}
\varepsilon_{x x}=\frac{1}{E_{x x}} \sigma_{x x}-\frac{v_{x y}}{E_{y y}} \sigma_{y y}, \\
\varepsilon_{y y}=-\frac{v_{y x}}{E_{x x}} \sigma_{x x}+\frac{1}{E_{y y}} \sigma_{y y} .
\end{gathered}
$$

The angle distortion is further given by

$$
2 \varepsilon_{x y}=\frac{1}{G_{x y}} \sigma_{x y} .
$$

Mechanical strain values (29) and (30) less than 0.02 and angle distortions (31) slighter than 0.22 radiant usually characterise the linear elastic mechanical problem $[8,11]$. Thus (29) can be approximated by the first derivate of the radial displacement

$$
\frac{\partial u}{\partial x}=\varepsilon_{x x}
$$

and (30) by the first derivate of the tangential displacement

$$
\frac{\partial v}{\partial y}=\varepsilon_{y y}
$$

The angle distortion (31) is further given by

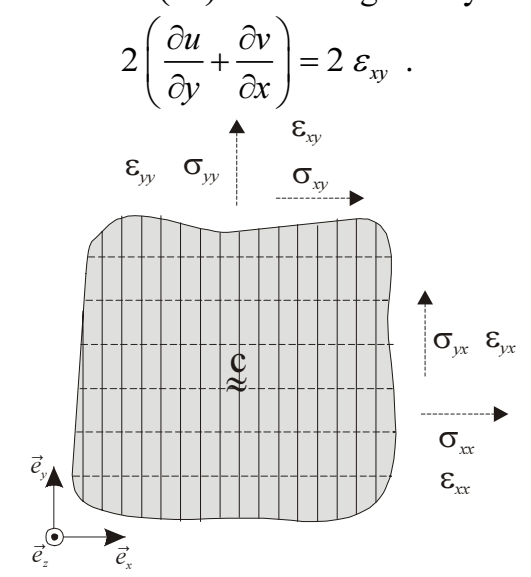

Fig. 3. Tensile and shear stress acting at the xy-plane in an anisotropic material.
Moreover, the identity

$$
\frac{v_{x y}}{E_{y y}}=\frac{v_{y x}}{E_{x x}}
$$

for orthotropic materials is implicit required due to the symmetric shear stress condition. Only four of the established five elastic constants from (29), (30) and (31) are independent from each other. The additionally introduced shear modulus

$$
G_{x y}=\frac{E_{x x} E_{y y}}{E_{x x}+E_{y y}+2 v_{x y} E_{y y}}
$$

reduces the number of unknown material coefficients to three [11]. The rewritten forms of (29), (30) and (31) yield to

$$
\begin{gathered}
\sigma_{x x}=\frac{1}{1-\left(v_{x y}\right)^{2} \frac{E_{x x}}{E_{y y}}}\left(E_{x x} \varepsilon_{x x}+v_{x y} E_{x x} \varepsilon_{y y}\right), \\
\sigma_{y y}=\frac{1}{1-\left(v_{x y}\right)^{2} \frac{E_{x x}}{E_{y y}}}\left(v_{x y} E_{x x} \varepsilon_{x x}+E_{y y} \varepsilon_{y y}\right), \\
\sigma_{x y}=2 \frac{E_{x x} E_{y y}}{E_{x x}+E_{y y}+2 v_{x y} E_{y y}} \varepsilon_{x y},
\end{gathered}
$$

whereby the coefficients of the symmetric material tensor (26) are identified as

$$
\begin{gathered}
c_{x x x x}=\frac{E_{x x}}{1-\left(v_{x y}\right)^{2} \frac{E_{x x}}{E_{y y}}}, \quad c_{x x y y}=\frac{v_{x y} E_{x x}}{1-\left(v_{x y}\right)^{2} \frac{E_{x x}}{E_{y y}}}, \\
c_{y y x x}=\frac{v_{y x} E_{y y}}{1-\left(v_{x y}\right)^{2} \frac{E_{x x}}{E_{y y}}}, \quad c_{y y y y}=\frac{E_{y y}}{1-\left(v_{x y}\right)^{2} \frac{E_{x x}}{E_{y y}}}, \\
c_{x y x y}=2 \frac{E_{x x} E_{y y}}{E_{x x}+E_{y y}+2 v_{x y} E_{y y}} .
\end{gathered}
$$

All other coefficients in (26) are disappearing in case of the considered mechanical two dimensional and linear orthotropic problem.

\section{Numerical investigations performed for orthotropic materials}

The reduction of the coupled electromechanical problem of fixed and current carrying Roebel bars to a simple electromagnetic problem as base of construction is not valid. Moreover, the local mechanical stress values at the slot wedge and the slot bottom are dominated by the amount of mechanical slot wedge fixing. Only the special case of loose Roebel bars in slots can be treated with a partial electromagnetic view of the problem [13].

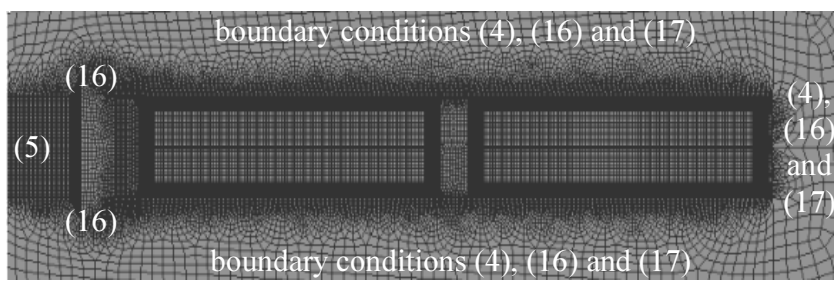

Fig. 4. The finite element mesh with electromagnetic and mechanical boundary conditions. 


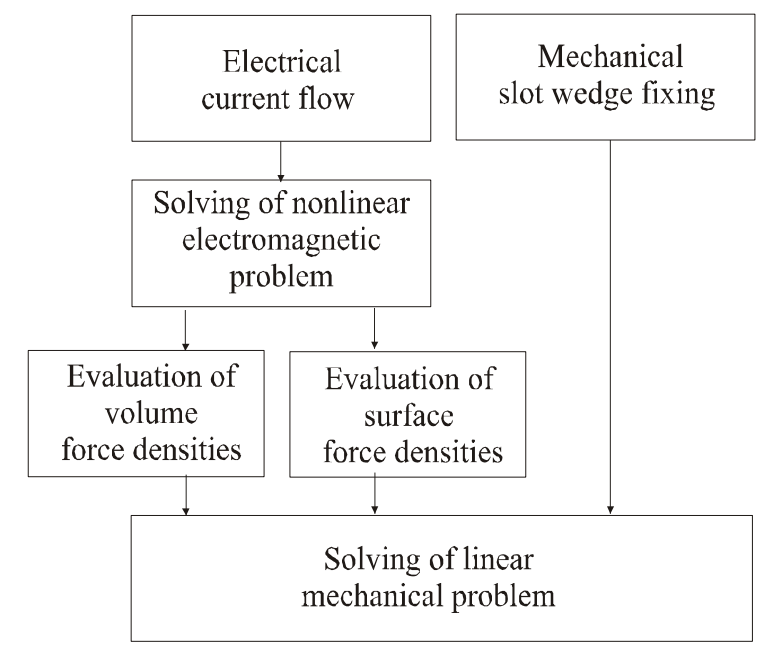

Fig. 5. Flow chart of the coupled numerical field calculation.

Some problems may arise within the air regions between e.g. airgap and slot wedge or the Roebel bar and the lateral slot wedge. Air regions mainly govern the electromagnetic field and they are therefore essential for the calculation. The numerically computation of the mechanical deformation does not take use of air regions due to the assumption of non deformity. They must be treated separately in the mechanical finite element calculation.

The necessary outer electromagnetic boundary condition (4) is applied parallel to the lateral iron slot surface as it is shown in Fig.4. In order to allow a normal flux density component near the airgap, the condition (5) is used.

In order to fix the mechanical iron slot pitch in space, vanishing displacement components (16) and (17) are applied. This assumption has no significant influence to the mechanical deformation behavior of materials which are embedded inside the slot because there are very tiny air layers between the inlaid parts and the lateral iron slot surfaces. The mechanical analysis uses default values (16) of the slot wedge fixing as it is presented in Fig.4.

The applied loads for the solution of the mechanical problem in Fig.5 are separately composed from electromagnetic and mechanical contributions. The acting volume force densities inside the conducting regions (6) and acting surface force densities (13) at the iron boundary of the slot are directly obtained from the nonlinear solutions of the electromagnetic analysis [12]. Whereas the mechanical loads are given by the default values of the wedge fixing (16). All numerical investigations on the mechanical behavior of the embedded materials inside the slot are based on a finite element implementation with (1) to (42).

The electromagnetic and in particular the mechanical material properties have a crucial influence to the invoked stress magnitudes. Various resins with or without unidirectional or orthogonal glass fiber reinforcement or even mats and woven fibers are used for building up each non-conducting anisotropic part of the structure shown in Fig.2. The same mechanical properties within all space directions only exist in the simple case of isotropic materials. Mechanical properties of the industrially used materials for building up the common structure presented in Fig.2 are given in Table II, III and IV.
TABLE II. - Material properties (1)

\begin{tabular}{lcccc}
\hline & $\begin{array}{c}E_{x x} \\
{\left[\mathrm{GN} / \mathrm{m}^{2}\right]}\end{array}$ & $\begin{array}{c}v_{x y} \\
{[1]}\end{array}$ & $\begin{array}{c}E_{y y} \\
{\left[\mathrm{GN} / \mathrm{m}^{2}\right]}\end{array}$ & $\begin{array}{c}v_{y x} \\
{[1]}\end{array}$ \\
\hline Iron (A) & 210,0 & 0,30 & 210,0 & 0,30 \\
Sub conductor (B) & 110,0 & 0,35 & 110,0 & 0,35 \\
Insulation (C) & 4,0 & 0,35 & 3,5 & 0,40 \\
Resin & 3,5 & 0,40 & 3,5 & 0,40 \\
Bar envelope (E) & 16,0 & 0,09 & 3,5 & 0,40 \\
Corona shielding (L) & 4,0 & 0,35 & 4,0 & 0,35 \\
Middle filler piece (D) & 5,0 & 0,24 & 3,0 & 0,40 \\
Slide band (J) & 7,0 & 0,17 & 3,0 & 0,40 \\
Bottom slot band (G) & 7,0 & 0,17 & 3,0 & 0,40 \\
Slot wedge (K) & 14,0 & 0,09 & 3,0 & 0,40 \\
Butt plate (H) & 7,0 & 0,17 & 3,0 & 0,40 \\
Slot covering spring (I) & 6,0 & 0,20 & 3,0 & 0,40 \\
Intermediary (F) & 11,0 & 0,11 & 3,0 & 0,40 \\
\hline
\end{tabular}

TABLE III. - Material properties (2)

\begin{tabular}{lcccc}
\hline & $\begin{array}{c}E_{x x} \\
{\left[\mathrm{GN} / \mathrm{m}^{2}\right]}\end{array}$ & $\begin{array}{c}v_{x y} \\
{[1]}\end{array}$ & $\begin{array}{c}E_{y y} \\
{\left[\mathrm{GN} / \mathrm{m}^{2}\right]}\end{array}$ & $\begin{array}{c}v_{y x} \\
{[1]}\end{array}$ \\
\hline Iron (A) & 210,0 & 0,30 & 210,0 & 0,30 \\
Sub conductor (B) & 110,0 & 0,35 & 110,0 & 0,35 \\
Insulation (C) & 18,0 & 0,09 & 4,0 & 0,40 \\
Resin & 4,0 & 0,40 & 4,0 & 0,40 \\
Bar envelope (E) & 60,0 & 0,03 & 4,0 & 0,40 \\
Corona shielding (L) & 4,0 & 0,35 & 4,0 & 0,35 \\
Middle filler piece (D) & 14,0 & 0,09 & 3,0 & 0,40 \\
Slide band (J) & 18,0 & 0,09 & 4,0 & 0,40 \\
Bottom slot band (G) & 14,0 & 0,09 & 3,0 & 0,40 \\
Slot wedge (K) & 25,0 & 0,06 & 4,0 & 0,40 \\
Butt plate (H) & 15,0 & 0,09 & 3,5 & 0,40 \\
Slot covering spring (I) & 17,0 & 0,08 & 3,5 & 0,40 \\
Intermediary (F) & 35,0 & 0,05 & 4,0 & 0,40 \\
\hline
\end{tabular}

TABLE IV. - Material properties (3)

\begin{tabular}{lcccc}
\hline & $\begin{array}{c}E_{x x} \\
{\left[\mathrm{GN} / \mathrm{m}^{2}\right]}\end{array}$ & $\begin{array}{c}v_{x y} \\
{[1]}\end{array}$ & $\begin{array}{c}E_{y y} \\
{\left[\mathrm{GN} / \mathrm{m}^{2}\right]}\end{array}$ & $\begin{array}{c}v_{y x} \\
{[1]}\end{array}$ \\
\hline Iron (A) & 210,0 & 0,30 & 210,0 & 0,30 \\
Sub conductor (B) & 110,0 & 0,35 & 110,0 & 0,35 \\
Insulation (C) & 12,0 & 0,02 & 0,59 & 0,32 \\
Resin & 4,0 & 0,40 & 4,0 & 0,40 \\
Bar envelope (E) & 150,0 & 0,01 & 4,0 & 0,40 \\
Corona shielding (L) & 4,0 & 0,35 & 4,0 & 0,35 \\
Middle filler piece (D) & 18,0 & 0,09 & 4,0 & 0,40 \\
Slide band (J) & 18,0 & 0,09 & 4,0 & 0,40 \\
Bottom slot band (G) & 18,0 & 0,09 & 4,0 & 0,40 \\
Slot wedge (K) & 35,0 & 0,04 & 3,5 & 0,40 \\
Butt plate (H) & 35,0 & 0,04 & 3,5 & 0,40 \\
Slot covering spring (I) & 35,0 & 0,04 & 3,5 & 0,40 \\
Intermediary (F) & 35,0 & 0,04 & 3,5 & 0,40 \\
\hline
\end{tabular}

\section{Analytically obtained solutions for the mechanical deformation problem}

\section{A. Nonlinear electromagnetic force densities}

Based on ideal permeable iron slot properties, the field distribution inside the slot has the ability to produce electromagnetic force densities in radial and tangential direction. Moreover, the influence of the analytical tangential force component in (11) to the deformation process inside the slot in case of in phase currents can be neglected [14]. Due to the rectangular slot geometry, the independency of the radial volume force components of upper and lower bar from the bar width is assumed. Thus, the radial volume force component of (11) is 
TABLE V. - Values for $\alpha_{k, l}$

\begin{tabular}{lccccc}
\hline & $\vartheta_{k, l}$ & & & $\chi_{k, l}$ & \\
& $k=I$ & $k=I I$ & & $k=I$ & $k=I I$ \\
$l=s$ & 1.021 & 0.681 & $l=s$ & 0.043 & -0.513 \\
$l=o$ & 1.001 & 0.977 & $l=o$ & 0.016 & -0.079 \\
\hline
\end{tabular}

TABLE VI. - Values for $\beta_{k, l}$

\begin{tabular}{lccccc}
\hline & $\gamma_{k, l}$ & & \multicolumn{3}{c}{$\lambda_{k, l}$} \\
& $k=I$ & $k=I I$ & & $k=I$ & $k=I I$ \\
$l=s$ & 0.766 & 0.896 & $l=s$ & -0.223 & -0.202 \\
$l=o$ & 0.981 & 0.988 & $l=o$ & -0.066 & -0.003 \\
\hline
\end{tabular}

$$
\begin{aligned}
f_{I, l}= & \mu_{0} \frac{I_{I, l}^{2}}{a b h}\left[\left(1-\alpha_{I, l}\right)+\beta_{I, l}\right]+ \\
& +\mu_{0} \frac{I_{I, l}^{2}}{a b h} \beta_{I, l}\left(1+\frac{h_{1}}{h}+\frac{h_{2}}{h}-\frac{x}{h}\right)
\end{aligned}
$$

in case of the lower bar I, $\mathrm{h}_{1}+\mathrm{h}_{2}+\mathrm{h}<\mathrm{x}<\mathrm{h}_{1}+\mathrm{h}_{2}+2 \mathrm{~h}$, and

$$
\begin{aligned}
f_{I I, l} & =\mu_{0} \frac{I_{I, l} I_{I I, l}}{a b h} \alpha_{I I, l}+ \\
& +\mu_{0} \frac{I_{I I, l}^{2}}{a b h} \beta_{I I, l}\left(1+\frac{h_{2}}{h}-\frac{x}{h}\right)
\end{aligned}
$$

inside the upper bar II, between $\mathrm{h}_{2}<\mathrm{x}<\mathrm{h}_{2}+\mathrm{h}$. The used indices 1 in (43) and (44) describes with $1=s$ the current flow of same direction and with $1=0$ the current flow of opposite direction. Saturation effects due to higher currents are approximated in (43) and (44) with

$$
\alpha_{k, l}=\vartheta_{k, l}\left(\frac{I_{k, l}}{I_{N}}\right)^{\chi_{k, l}}
$$

and

$$
\beta_{k, l}=\gamma_{k, l}\left(\frac{I_{k, l}}{I_{N}}\right)^{\lambda_{k, l}}
$$

The typical ratio of slot height and slot width restricts the coefficients (45) and (46) to characteristic courses. They are summarized in Table $\mathrm{V}$ and Table VI, whereby a tolerance of $2 \%$ should be considered.

\section{B. Mechanical stress caused by bar fixing and electrical current flow}

In case of fixed bars inside the slot, the boundary condition has to include the necessary wedge displacement (16) due to the default value $\mathrm{u}_{2}$ which is applied at the position $\mathrm{x}=\mathrm{h}_{2}-\mathrm{m}-\mathrm{n}$ of Fig.2. The assumption of a very weak and therefore negligible deformation of the slot wedge leads to the boundary condition

$$
\left.u_{I I, l}\right|_{x=h_{2}, y}=u_{2} \text {. }
$$

The slot bottom is assumed to be rigid

$$
\left.u_{I, l}\right|_{x=h_{1}+h_{2}+2 h, y}=0
$$

for all analytical investigations. Moreover, the steady state condition of the mechanical stress

$$
\left.\sigma_{x x, I I, l}\right|_{x=h_{2}+h, y}=\left.\sigma_{x x, I, I}\right|_{x=h_{1}+h_{2}+h, y},
$$
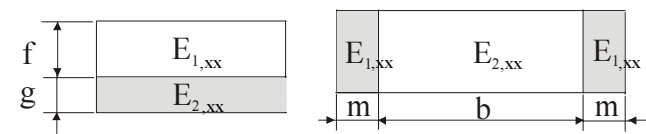

Fig. 6. Modulus of elasticity for serial and parallel layers.

as well as the mechanical displacement

$$
\left.u_{I I, l}\right|_{x=h_{2}+h, y}=\left.u_{I, l}\right|_{x=h_{1}+h_{2}+h, y},
$$

neglects the deformation of the intermediary part.

Moreover, the analytically description of the complex mechanical behavior of the considered body requires a homogenous modulus of elasticity for each investigated material assembly which is given in Table II, III and IV.

The unidirectional acting forces of electromagnetic and mechanical origin allow the identification of a common modulus of elasticity only from a subset of the totally mechanical material properties.

The material structure, even the serial or the parallel one in Fig.6, can be described by two separate moduli of elasticity and the according geometric dimensions. Each common structure in Fig. 6 reacts to a default mechanical pressure, which is applied at both horizontal boundaries, as a material characterized by the modified modulus

$$
E_{x x}^{=}=\left(1+\frac{f}{g}\right) \frac{E_{2, x x}}{\left(1+\frac{f}{g} \frac{E_{2, x x}}{E_{1, x x}}\right)}
$$

in case of a the serial structure, and with

$$
E_{x x}^{\|}=E_{1, x x} \frac{2 m}{(2 m+b)}+E_{2, x x} \frac{b}{(2 m+b)}
$$

in case of the parallel structure.

Thus, the serial layer of conductor and insulation in Fig.1 can be globally described with the geometric ratio $\frac{\mathrm{f}}{\mathrm{g}}=10$ and (51). The according values are given in Table VII for each investigated material assembly.

The usage of the geometric dimensions $2 \cdot \mathrm{m}=0,0062$ and $b=0,0159$ allows to combine the parallel layer of subconductor with insulation and the lateral bar envelope with (52) for each material arrangement. The common elasticity values are included in Table VIII.

The consideration of the horizontal bar envelope situated under and above the conducting region leads with the geometric proportion $\frac{\mathrm{f}}{\mathrm{g}}=\frac{0,0709}{2 \cdot 0,0031}=11,4$ and (51) to the common values in Table IX for each material assembly.

TABLE VII. - Modulus of elasticity for conductors and insulation

\begin{tabular}{ccccc}
\hline Assembly & & (1) & (2) & (3) \\
\hline$E_{I, x x}$ & {$\left[\mathrm{GN} / \mathrm{m}^{2}\right]$} & 110,0 & 110,0 & 110,0 \\
$E_{2, x x}$ & {$\left[\mathrm{GN} / \mathrm{m}^{2}\right]$} & 4,0 & 18,0 & 12,0 \\
$E_{x x}^{-}$ & {$\left[\mathrm{GN} / \mathrm{m}^{2}\right]$} & 32,3 & 75,1 & 63,1 \\
\hline
\end{tabular}

TABLE VIII. - Modulus of elasticity regarding the bar envelope

\begin{tabular}{ccccc}
\hline Assembly & & (1) & (2) & (3) \\
\hline$E_{l, x x}$ & {$\left[\mathrm{GN} / \mathrm{m}^{2}\right]$} & 16,0 & 60,0 & 150,0 \\
$E_{2, x x}$ & {$\left[\mathrm{GN} / \mathrm{m}^{2}\right]$} & 32,3 & 75,1 & 63,1 \\
$E_{x x}^{\|}$ & {$\left[\mathrm{GN} / \mathrm{m}^{2}\right]$} & 27,7 & 70,9 & 87,5 \\
\hline
\end{tabular}


TABLE IX. - Modulus of elasticity regarding the bar envelope upside and down the conductors

\begin{tabular}{ccccc}
\hline Assembly & & (1) & (2) & (3) \\
\hline$E_{l, x x}$ & {$\left[\mathrm{GN} / \mathrm{m}^{2}\right]$} & 27,7 & 70,9 & 87,5 \\
$E_{2, x x}$ & {$\left[\mathrm{GN} / \mathrm{m}^{2}\right]$} & 16,0 & 60,0 & 150,0 \\
$E_{x x}^{\|}$ & {$\left[\mathrm{GN} / \mathrm{m}^{2}\right]$} & 26,1 & 69,8 & 90,5 \\
\hline
\end{tabular}

TABLE X. - Modulus of elasticity regarding the intermediary part

\begin{tabular}{ccccc}
\hline Assembly & & (1) & (2) & (3) \\
\hline$E_{1, x x}$ & {$\left[\mathrm{GN} / \mathrm{m}^{2}\right]$} & 26,1 & 69,8 & 90,5 \\
$E_{2, x x}$ & {$\left[\mathrm{GN} / \mathrm{m}^{2}\right]$} & 11,0 & 35,0 & 35,0 \\
$E_{x x}$ & {$\left[\mathrm{GN} / \mathrm{m}^{2}\right]$} & 24,2 & 66,1 & 82,9 \\
\hline
\end{tabular}

The common modulus of elasticity including the structural layer of the intermediary part is obtained with $\frac{\mathrm{f}}{\mathrm{g}}=\frac{2 \cdot 0,0709+4 \cdot 0,0031}{0,0155-2 \cdot 0,0031}=16,4$ from (51). Values are further given

in Table $\mathrm{X}$ for each considered arrangement.

The high ratio of geometric length and width of the homogenous conducting areas prevent any warping across the common bar width according to Bernoulli's theorem [15]. Consequently, the condition of a vanishing angle distortion (31) in both bars is valid. The tensile stress (15) is given inside the upper bar $\mathrm{h}_{2}<\mathrm{x}<\mathrm{h}_{2}+\mathrm{h}$ as

$$
\begin{aligned}
& \sigma_{x x, I I, l}=-\frac{1}{2} u_{2} k_{1} k_{2} \frac{E}{h}+ \\
& +\frac{\mu_{0}}{a b} k_{2}\left\{I_{I} I_{I I} \alpha_{I I, l}\left[\frac{3}{4}-\left(\frac{x}{h}-\frac{h_{2}}{h}\right)\right]+\right. \\
& +I_{I I}^{2} \beta_{I I, l}\left[\frac{5}{12}-\left(\frac{x}{h}-\frac{h_{2}}{h}\right)+\frac{1}{2}\left(\frac{x}{h}-\frac{h_{2}}{h}\right)^{2}\right]+ \\
& \left.+I_{I}^{2}\left[\frac{1}{4}\left[\left(1-\alpha_{I, l}\right)+\beta_{I, l}\right]-\frac{1}{12} \beta_{I, l}\right]\right\}
\end{aligned}
$$

and inside the lower bar $\mathrm{h}_{1}+\mathrm{h}_{2}+\mathrm{h}<\mathrm{x}<\mathrm{h}_{1}+\mathrm{h}_{2}+2 \mathrm{~h}$ as

$$
\begin{aligned}
& \sigma_{x x, I, l}=-\frac{1}{2} u_{2} k_{1} k_{2} \frac{E}{h}+ \\
& +\frac{\mu_{0}}{a b} k_{2}\left\{I_{I}^{2} \frac{1}{4}\left[\left(1-\alpha_{I, l}\right)+\beta_{I, l}\right]-\right. \\
& -I_{I}^{2} \frac{1}{2}\left[\left(1-\alpha_{I, l}\right)+\beta_{I, l}\right]\left(\frac{x}{h}-\frac{h_{1}}{h}-\frac{h_{2}}{h}-1\right)+ \\
& +I_{I}{ }^{2} \frac{1}{2} \beta_{I, l}\left(\frac{x}{h}-\frac{h_{1}}{h}-\frac{h_{2}}{h}-1\right)^{2}-I_{I}^{2} \frac{1}{12} \beta_{I, l}- \\
& \left.-I_{I} I_{I I} \alpha_{I I, l}\left(\frac{1}{4}\right)-I_{I I}{ }^{2} \beta_{I I, l}\left(\frac{1}{12}\right)\right\}
\end{aligned}
$$

The nonlinear electromagnetic correction coefficients (45) and (46) are chosen in dependency on the direction $\mathrm{l}=\mathrm{s}, \mathrm{O}$ of both electrical bar currents. The analytically derived expressions (53) and (54) are only suitable to describe average values of the existing stress along the homogenous bar width.

\begin{tabular}{|c|c|c|c|c|}
\hline Assembly & & (1) & (2) & (3) \\
\hline$\left.\sigma_{x x, I, s}{ }^{m u m}\right|_{x=h 1+h 2+2 h, y}$ & {$\left[\mathrm{MN} / \mathrm{m}^{2}\right]$} & 6.58 & 15.35 & 17.77 \\
\hline$\left.\sigma_{x x, I, S}^{\text {anal }}\right|_{x=h 1+h 2+2 h, y}$ & {$\left[\mathrm{MN} / \mathrm{m}^{2}\right]$} & 7.56 & 15.08 & 17.53 \\
\hline$\left.\sigma_{x x, I I, s}{ }^{m u m}\right|_{x=h 2, y}$ & {$\left[\mathrm{MN} / \mathrm{m}^{2}\right]$} & 2.16 & 10.94 & 13.35 \\
\hline$\sigma_{x x, I I, s}$ anal $_{x=h 2, y}$ & {$\left[\mathrm{MN} / \mathrm{m}^{2}\right]$} & 3.25 & 10.71 & 13.13 \\
\hline
\end{tabular}

Moreover, the coefficient $\mathrm{k}_{1}$ of Table XI enables us to include various mechanical material assemblies in the analytical solutions (53) and (54).

\begin{tabular}{|c|c|c|c|c|}
\hline Assembly & & (1) & (2) & (3) \\
\hline$\left.\sigma_{x x, I, o}^{m u m}\right|_{x=h 1+h 2+2 h, y}$ & {$\left[\mathrm{MN} / \mathrm{m}^{2}\right]$} & 5.08 & 13.78 & 15.7 \\
\hline$\sigma_{x x, I, o}$ anal $\left.\right|_{x=h 1+h 2+2 h, y}$ & {$\left[\mathrm{MN} / \mathrm{m}^{2}\right]$} & 4.39 & 12.72 & 15.63 \\
\hline$\left.\sigma_{x x, I I, o}{ }^{n u m}\right|_{x=h 2, y}$ & {$\left[\mathrm{MN} / \mathrm{m}^{2}\right]$} & 4.96 & 13.66 & 15.84 \\
\hline$\left.\sigma_{x x, I I, o}{ }^{\text {anal }}\right|_{x=h 2, y}$ & {$\left[\mathrm{MN} / \mathrm{m}^{2}\right]$} & 4.37 & 12.64 & 15.52 \\
\hline
\end{tabular}

TABLE XI. - Mechanical correction coefficients for the material properties and dimensions

\begin{tabular}{ccccc}
\hline Assembly & & (1) & (2) & (3) \\
\hline$k_{1}=\frac{E_{x x}^{\|}}{E}$ & {$[\%]$} & 22.1 & 60,0 & 75,4 \\
$k_{2}=\frac{b}{a}$ & {$[\%]$} & 72.3 & 72.3 & 72.3 \\
\hline
\end{tabular}

TABLE XII. - Numerically and analytically calculated values for $\mathrm{u}_{2}=4.210^{-5} \mathrm{~m}$ and currents $15 \mathrm{p} . \mathrm{u}$ of same phase

TABLE XIII. - Numerically and analytically calculated values for $\mathrm{u}_{2}=4.210^{-5} \mathrm{~m}$ and currents $7,5 \mathrm{p}$.u of opposite phase

The reference value for the modulus of elasticity in the Table XI is $E=110\left[\mathrm{GN} / \mathrm{m}^{2}\right]$. Differences between the width of the Roebel bar and the conducting domains are regarded by the coefficient $\mathrm{k}_{2}$.

The calculated average values of mechanical stress, both for currents with same direction in Table XII and for currents with opposite direction in Table XIII are obtained from (53) and (54) respectively. The local mechanical stress significantly depends on the actual slot wedge fixing [16]. A stronger slot wedge fixing causes a higher deformation of the inlaid materials.

Moreover, the comparison of the calculated mean values of the mechanical stress shows in most cases the good agreement between the numerical and analytical method.

\section{Regarding mechanical stress peaks}

The local rising stress values at the slot bottom and the slot wedge are not directly accessible in (53) and (54). Concerning the material abrasion an improvement in (53) and (54) is required in order to regard local rising stress magnitudes correctly. With the assumption of a non deformable slot bottom, the stress distribution at the interface of bar and slot bottom is assumed as

$$
\sigma_{x x, k, l}^{\prime}=\sigma_{x x, k, l} \frac{2}{\pi} \frac{1}{\sqrt{1-\left(y \cdot \frac{2}{a}-1\right)^{2}}},
$$

whereby the average stress values (53), (54) and the geometric width $y=0 . . . \mathrm{a}$ are used [17]. The relationship (55) enables us to determine the ratio of the maximal stress increase analytically in relation to the mean value at $5 \%$ or due to symmetry conditions even at $95 \%$ of the considered length to

$$
\frac{\sigma_{x x, k, l}^{\prime}}{\sigma_{x x, k, l}}=\frac{2}{\pi} \frac{1}{\sqrt{1-0.9^{2}}}=146 \% \text {. }
$$

This imposingly shows the basic necessity of regarding the occurring stress peaks in the analytical calculation. 


\section{Local raising mechanical stress along the slot bottom of restrained Roebel bars}

Analytical mechanical stress courses calculated with (55) are depicted in Fig.7, Fig.8 and Fig.9 along the slot bottom of Fig.2 for several different material assemblies.

Two vertically lines indicate the transfer from copper and insulation region to the envelope inside the Roebel bar. Thus, we can realize that the stress starts to rise up significantly near the lateral envelope in each case.

From Fig.7 we obtain especially at the slot bottom lower numerically calculated stress peaks in comparison to the analytically approach. The strong deviation is caused by the weak material properties, which do not exactly fulfill the necessary analytical preconditions (47) and (50).

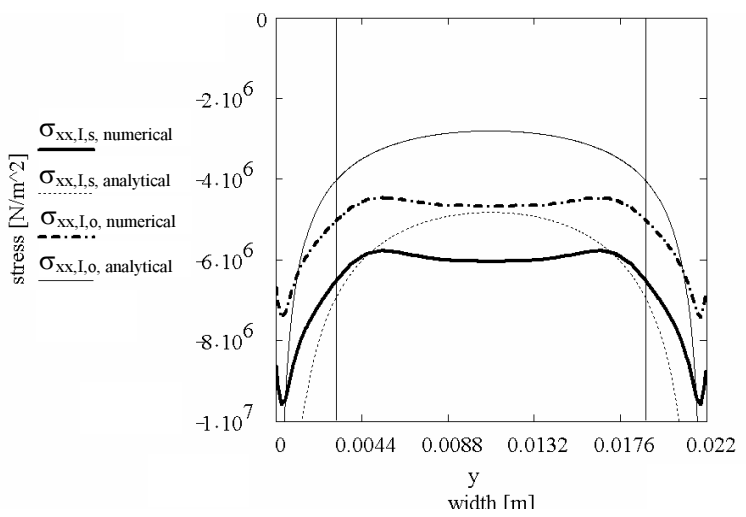

Fig. 7. Analytical and numerical stress courses for assembly (1) depicted along the slot bottom for $\mathrm{u}_{2}$ fixing and currents of both directions versus bar width.

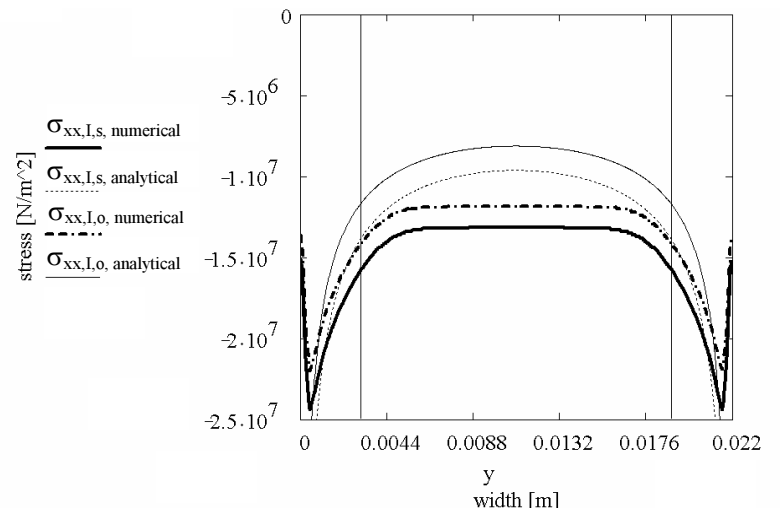

Fig. 8. Analytical and numerical stress courses for assembly (2) depicted along the slot bottom for $\mathrm{u}_{2}$ fixing and currents of both directions versus bar width.

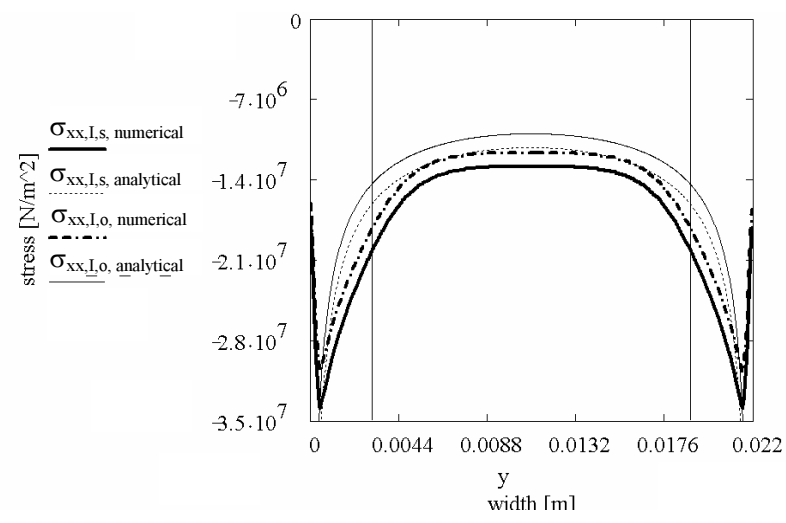

Fig. 9. Analytical and numerical stress courses for assembly (3) depicted along the slot bottom for $\mathrm{u}_{2}$ fixing and currents of both directions versus bar width.
The best agreement between the numerical and analytical projection is given for Fig.8 and Fig.9. Shape and absolute stress peaks agree largely. Used materials with high elasticity modulus further fulfill the explained requirement for (55) in an acceptable way.

By keeping the mechanical fixing and the magnitude of the electrical current of same phase constant, we can observe from a comparison of the considered material assembly in Fig.7 and Fig.9 that the maximal stress peak rises up to about $350 \%$.

The numerically obtained mechanical stress peaks at the material edges of the Roebel bar can be analytically approximated by values of (55) at locations between $90 \%$ and $95 \%$ of the bar width with satisfactory correctness.

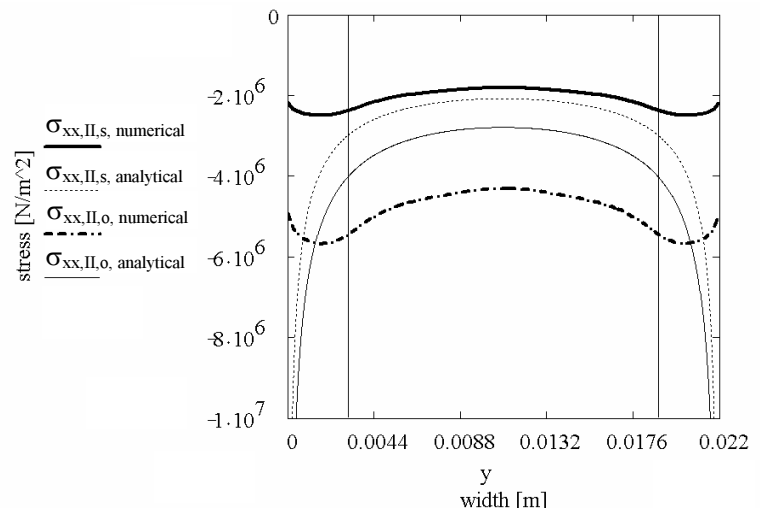

Fig. 10. Analytical and numerical stress courses for assembly (1) depicted along the slot wedge for $\mathrm{u}_{2}$ fixing and currents of both directions versus bar width.

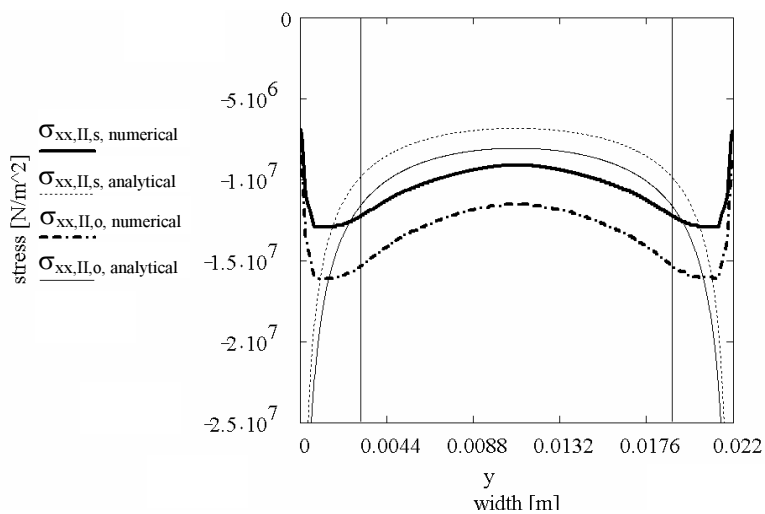

Fig. 11. Analytical and numerical stress courses for assembly (2) depicted along the slot wedge for $\mathrm{u}_{2}$ fixing and currents of both directions versus bar width.

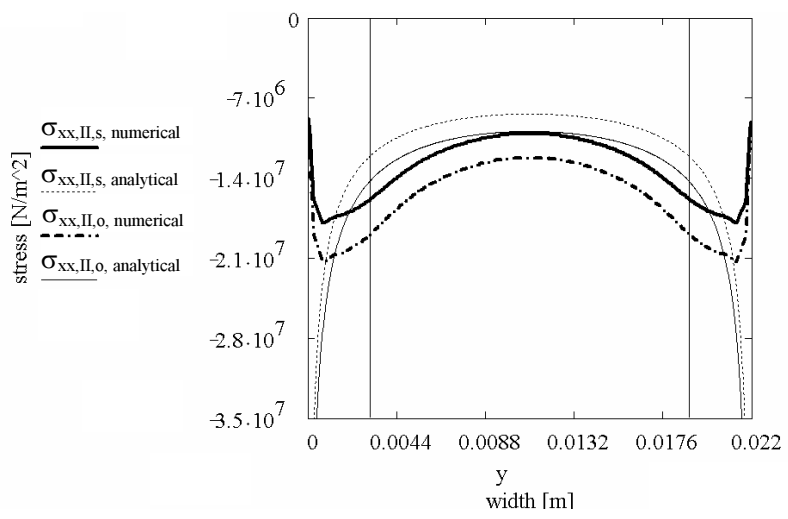

Fig. 12. Analytical and numerical stress courses for assembly (3) depicted along the slot wedge for $\mathrm{u}_{2}$ fixing and currents of both directions versus bar width. 


\section{Local raising mechanical stress along the slot wedge of restrained Roebel bars}

The main problem within the analytical approach arises within the finite thickness of the slot wedge. Hence, the material characteristic fulfills the requirement for (47) or (55) in dependency on the investigated material assembly only approximately. Comparisons of analytical stress courses (55) calculated from mean values of Table XII and Table XIII and obtained numerical results are given in Fig.10, Fig. 11 and Fig. 12.

The numerically calculated distributions in Fig.10, Fig.11 and Fig. 12 deliver reduced stress peaks in comparison to the analytical courses due to the regarded deformation of the slot wedge. Furthermore, currents of same phase avoid those slot wedge deformation and yield to a better agreement between numerical and analytical courses, as currents of opposite phase do.

\section{Conclusions}

The mechanical stress distribution at the slot wedge and the slot bottom of a synchronous generator is analyzed for a large class of utilized industrial materials by analytical and numerical methods.

Nonlinear magnetic saturation effects are included in the performed analytical force density calculation due to correction coefficients. Thereby, the different influences of the electrical phase shift of the bar currents are further explained. Moreover, the mechanical properties are regarded in the analytical approach by a certain kind of homogenization technique for anisotropic composite materials. Special attention is further given to the inclusion of locally occurring mechanical stress peaks in the analytical obtained stress especially at the slot bottom and the slot wedge.

The validity of the derived analytical expressions is given by a detailed comparison with numerically obtained results. The good agreement between both presented methods is shown for several cases.

\section{References}

[1] H. Sequenz, Die Herstellung der Wicklungen elektrischer Maschinen. Springer, Wien/New York (1973).

[2] Sommerfeld, Elektrodynamik. Akademische Verlagsgesellschaft, Leipzig (1964).

[3] L. D. Landau, E. M. Lifschitz, Lehrbuch der theoretischen Physik. Akademie Verlag, Berlin (1985).

[4] R. M. Fano, J. J. Chu, R. B. Adler, Electromagnetic Fields, Energy and Forces. John Wiley \& Sons, London (1960).

[5] H. Woodson, J. R. Melcher, Electromechanical Dynamics. John Wiley \& Sons, London (1968).

[6] F. C. Moon, Magneto Solid Mechanics. John Wiley \& Sons, London (1939).

[7] W. F. Brown, Magnetoelastic Interactions. Springer, Berlin/ Heidelberg (1966).

[8] G. A. Holzapfel, Nonlinear Solid Mechanics. John Wiley \& Sons, London (2000).

[9] H. Parkus, Mechanik der festen Körper, Springer, Wien/ New York (1988).

[10]G. Grabner, E. Schmidt, "Numerical Computation of Electromagnetic Force Densities along the Iron Boundaries Regarding Magnetic Saturation," Proc. IGTE 2002.

[11]S. G. Lekhnitzskii, Theory of Elasticity of an Anisotropic Body. Mir Publisher, Moscow (1981).

[12]C. Grabner, J. Bacher and E. Schmidt, "The effect of Electromagnetic and Mechanical Forces on Single Items used in Electrical Machines," in Proc. PIERS 2002.

[13]K. Vogt, Berechnung rotierender elektrischer Maschinen, Verlag Technik, Berlin (1988).

[14]C. Grabner, J. Bacher and H. Koefler, " The Mechanical Consequences of Radial and Tangential Volume Forces in Loose Roebel Bars of Large Synchronous Machines," in Proc. UPEC 2001

[15]A. Föppl, Vorlesungen über technische Mechanik. Oldenburg, München/Berlin (1943).

[16]C. Grabner, E. Schmidt, "Analysis of the Coupled Electromagnetic and Mechanical Problem arising with the Radial Slot Wedge Fixing of Slot Conductors in Large Electrical Machines," in Proc. CEM 2002.

[17]I. Szabó, Höhere Technische Mechanik. Springer, Berlin/ Heidelberg/New York (1972). 